\title{
Composition operators in hyperbolic general Besov-type spaces
}

\author{
A. El-Sayed Ahmed ${ }^{1,2}$ \\ ${ }^{1}$ Sohag University, Faculty of Science, \\ Department of Mathematics, \\ 82524 Sohag, Egypt. \\ ${ }^{2}$ Taif University, \\ Faculty of Science, Mathematics, \\ M. A. BAKHIT \\ Department of Mathematics, \\ Faculty of Science, Assiut Branch, \\ Al-Azhar University, Assiut 32861, Egypt. \\ mabakhit2007@hotmail.com \\ Department, box 888 El-Hawiyah, El-Taif 5700, \\ Saudi Arabia. \\ ahsayed80@hotmail.com
}

\begin{abstract}
In this paper we introduce natural metrics in the hyperbolic $\alpha$-Bloch and hyperbolic general Besov-type classes $F^{*}(p, q, s)$. These classes are shown to be complete metric spaces with respect to the corresponding metrics. Moreover, compact composition operators $C_{\phi}$ acting from the hyperbolic $\alpha$-Bloch class to the class $F^{*}(p, q, s)$ are characterized by conditions depending on an analytic self-map $\phi: \mathbb{D} \rightarrow \mathbb{D}$.
\end{abstract}

\section{RESUMEN}

En este artículo introducimos una métrica natural en las clases hiperbólicas $\alpha$-Bloch y tipo Besov generales. Estas clases se muestra que son espacios métricos completos respecto de las métricas correspondientes. Además se caracterizan los operadores de composición compactos $C_{\phi}$ que actúan desde las clases hiperbólicas $\alpha$-Bloch en la clase $F^{*}(p, q, s)$ por condiciones que dependen de la autoaplicación analítica $\phi: \mathbb{D} \rightarrow \mathbb{D}$.

Keywords and Phrases: Hyperbolic classes, composition operators, Lipschitz continuous, $\alpha$ Bloch space, $F^{*}(p, q, s)$ class.

2010 AMS Mathematics Subject Classification: 47B38, 30D50, 30D45, 46E15. 


\section{Introduction}

Let $\mathbb{D}:=\{z \in \mathbb{C}:|z|<1\}$ be the open unit disc of the complex plane $\mathbb{C}, \partial \mathbb{D}$ it's boundary. Let $\mathcal{H}(\mathbb{D})$ denote the space of all analytic functions in $\mathbb{D}$ and let $\mathrm{B}(\mathbb{D})$ be the subset of $\mathcal{H}(\mathbb{D})$ consisting of those $f \in \mathcal{H}(\mathbb{D})$ for which $|\mathrm{f}(z)|<1$ for all $z \in \mathbb{D}$. Also, $\mathrm{dA}(z)$ be the normalized area measure on $\mathbb{D}$ so that $A(\mathbb{D}) \equiv 1$.

Let the Green's function of $\mathbb{D}$ be defined as $g(z, a)=\log \frac{1}{\left|\varphi_{a}(z)\right|}$, where $\varphi_{a}(z)=\frac{a-z}{1-\bar{a} z}$, for $z, a \in \mathbb{D}$ is the Möbius transformation related to the point $a \in \mathbb{D}$.

If $(X, d)$ is a metric space, we denote the open and closed balls with center $x$ and radius $r>0$ by $B(x, r):=\{y \in X: d(y, x)<r\}$ and $\bar{B}(x, r):=\{y \in X: d(x, y) \leq r\}$, respectively.

Hyperbolic function classes are usually defined by using either the hyperbolic derivative $f^{*}(z)=$ $\frac{\left|f^{\prime}(z)\right|}{1-|f(z)|^{2}}$ of $f \in B(\mathbb{D})$, or the hyperbolic distance $\rho(f(z), 0):=\frac{1}{2} \log \left(\frac{1+|f(z)|}{1-|f(z)|}\right)$ between $f(z)$ and zero.

A function $f \in B(\mathbb{D})$ is said to belong to the hyperbolic $\alpha$-Bloch class $\mathcal{B}_{\alpha}^{*}$ if

$$
\|f\|_{\mathcal{B}_{\alpha}^{*}}=\sup _{z \in \mathbb{D}} f^{*}(z)\left(1-|z|^{2}\right)^{\alpha}<\infty,
$$

The little hyperbolic Bloch-type class $\mathcal{B}_{\alpha, 0}^{*}$ consists of all $f \in \mathcal{B}_{\alpha}^{*}$ such that

$$
\lim _{|z| \rightarrow 1} f^{*}(z)\left(1-|z|^{2}\right)^{\alpha}=0 .
$$

The usual $\alpha$-Bloch spaces $\mathcal{B}_{\alpha}$ and $\mathcal{B}_{\alpha, 0}$ are defined as the sets of those $f \in \mathcal{H}(\mathbb{D})$ for which

$$
\|f\|_{\mathcal{B}_{\alpha}}=\sup _{z \in \mathbb{D}}\left|f^{\prime}(z)\right|\left(1-|z|^{2}\right)^{\alpha}<\infty
$$

and

$$
\lim _{|z| \rightarrow 1}\left|f^{\prime}(z)\right|\left(1-|z|^{2}\right)^{\alpha}=0
$$

respectively.

It is obvious that $\mathcal{B}_{\alpha}^{*}$ is not a linear space since the sum of two functions in $B(\mathbb{D})$ does not necessarily belong to $\mathrm{B}(\mathbb{D})$.

We now turn to consider hyperbolic $F(p, q, s)$ type classes, which will be called $F^{*}(p, q, s)$. For $0<p, s<\infty,-2<q<\infty$, the hyperbolic class $F^{*}(p, q, s)$ consists of those functions $f \in B(\mathbb{D})$ for which (see [7])

$$
\|f\|_{\mathrm{F}^{*}(\mathbf{p}, \mathbf{q}, \mathrm{s})}^{\mathrm{p}}=\sup _{\mathbf{a} \in \mathbb{D}} \int_{\mathbb{D}}\left(f^{*}(z)\right)^{\mathrm{p}}\left(1-|z|^{2}\right)^{\mathfrak{q}} \mathrm{g}^{\mathrm{s}}(z, \mathfrak{a}) \mathrm{dA}(z)<\infty .
$$

Moreover, we say that $f \in F^{*}(p, q, s)$ belongs to the class $F_{0}^{*}(p, q, s)$ if

$$
\lim _{|a| \rightarrow 1} \int_{\mathbb{D}}\left(f^{*}(z)\right)^{p}\left(1-|z|^{2}\right)^{q} g^{s}(z, a) d A(z)=0 .
$$


The usual general Besov-type spaces $F(p, q, s)$ (defined using the conventional derivative $f^{\prime}$ instead of $f^{*}$ ) and their norms are denoted by the same symbols but with $f^{\prime}$.

Yamashita was probably the first one considered systematically hyperbolic function classes. He introduced and studied hyperbolic Hardy, BMOA and Dirichlet classes in [14, 15, 16] and others. More recently, Smith studied inner functions in the hyperbolic little Bloch-class [11, and the hyperbolic counterparts of the $\mathrm{Q}_{p}$ spaces were studied by $\mathrm{Li}$ in $[7$ and $\mathrm{Li}$ et. al. in [8]. Further, hyperbolic $Q_{p}$ classes and composition operators studied by Pérez-González et. al. in [10]. Very recently the first author in 1, gave some characterizations of hyperbolic $Q(p, \alpha)$ classes and the hyperbolic $(p, \alpha)$-Bloch classes by composition operators.

In this paper we will study the hyperbolic $\alpha$-Bloch classes $\mathcal{B}_{\alpha}^{*}$ and the general hyperbolic $F^{*}(p, q, s)$ type classes. We will also give some results to characterize Lipschitz continuous and compact composition operators mapping from the hyperbolic $\alpha$-Bloch class $\mathcal{B}_{\alpha}^{*}$ to $F^{*}(p, q, s)$ class by conditions depending on the symbol $\phi$ only.

Note that the general hyperbolic $F^{*}(p, q, s)$ type classes include the class of so-called $Q_{p}^{*}$ classes and the class of (hyperbolic) Besov class $\mathrm{B}_{\mathrm{p}}^{*}$. Thus, the results are generalizations of the recent results of Pérez-González, Rättyä and Taskinen [10].

For any holomorphic self-mapping $\phi$ of $\mathbb{D}$. The symbol $\phi$ induces a linear composition operator $C_{\phi}(f)=f \circ \phi$ from $\mathcal{H}(\mathbb{D})$ or $B(\mathbb{D})$ into itself. The study of composition operator $C_{\phi}$ acting on spaces of analytic functions has engaged many analysts for many years (see e.g. [2, 3, 4, 5, 8, 9, 17 , and others).

Recall that a linear operator $T: X \rightarrow Y$ is said to be bounded if there exists a constant $C>0$ such that $\|T(f)\|_{Y} \leq C\|f\|_{X}$ for all maps $f \in X$. By elementary functional analysis, a linear operator between normed spaces is bounded if and only if it is continuous, and the boundedness is trivially also equivalent to the Lipschitz-continuity. Moreover, $T: X \rightarrow Y$ is said to be compact if it takes bounded sets in $X$ to sets in $Y$ which have compact closure. For Banach spaces $X$ and $Y$ contained in $B(\mathbb{D})$ or $\mathcal{H}(\mathbb{D}), T: X \rightarrow Y$ is compact if and only if for each bounded sequence $\left\{x_{n}\right\} \in X$, the sequence $\left\{T x_{n}\right\} \in Y$ contains a subsequence converging to a function $f \in Y$.

Definition 1.1. A composition operator $\mathrm{C}_{\phi}: \mathcal{B}_{\alpha}^{*} \rightarrow \mathrm{F}^{*}(\mathrm{p}, \mathrm{q}, \mathrm{s})$ is said to be bounded, if there is a positive constant $\mathrm{C}$ such that $\left\|\mathrm{C}_{\phi} \mathrm{f}\right\|_{\mathrm{F} *(\mathfrak{p}, \mathrm{q}, \mathrm{s})} \leq \mathrm{C}\|\mathrm{f}\|_{\mathcal{B}_{\alpha}^{*}}$ for all $\mathrm{f} \in \mathcal{B}_{\alpha}^{*}$.

Definition 1.2. A composition operator $\mathrm{C}_{\phi}: \mathcal{B}_{\alpha}^{*} \rightarrow \mathrm{F}^{*}(\mathrm{p}, \mathrm{q}, \mathrm{s})$ is said to be compact, if it maps any ball in $\mathcal{B}_{\alpha}^{*}$ onto a precompact set in $\mathrm{F}^{*}(\mathrm{p}, \mathrm{q}, \mathrm{s})$.

The following lemma follows by standard arguments similar to those outline in Lemma 3.8 of [12. Hence we omit the proof.

Lemma 1.3. Assume $\phi$ is a holomorphic mapping from $\mathbb{D}$ into itself. Let $0<p, s<\infty,-1<$ 
$\mathrm{q}<\infty$ and $0<\alpha<\infty$. Then $\mathrm{C}_{\mathrm{\phi}}: \mathcal{B}_{\alpha}^{*} \rightarrow \mathrm{F}^{*}(\mathrm{p}, \mathrm{q}, \mathrm{s})$ is compact if and only if for any bounded sequence $\left\{f_{\mathfrak{n}}\right\}_{\mathfrak{n} \in \mathbb{N}} \in \mathcal{B}_{\alpha}^{*}$ which converges to zero uniformly on compact subsets of $\mathbb{D}$ as $n \rightarrow \infty$, we have $\lim _{n \rightarrow \infty}\left\|C_{\phi} f_{n}\right\|_{F^{*}(p, q, s)}=0$.

The following lemma can be found in [6], Theorem 2.1.1.

Lemma 1.4. Let $0<\alpha<\infty$, then there exist two holomorphic maps $\mathrm{f}, \mathrm{g}: \mathbb{D} \rightarrow \mathbb{C}$ such that for some constant $\mathrm{C}$,

$$
\left(f^{\prime}(z)+g^{\prime}(z)\right)\left(1-|z|^{2}\right)^{\alpha} \geq C>0, \quad \text { for each } z \in \mathbb{D} .
$$

\section{Hyperbolic classes and natural metrics}

In this section we introduce natural metrics on the hyperbolic $\alpha$-Bloch classes $\mathcal{B}_{\alpha}^{*}$ and the classes $F^{*}(p, q, s)$.

Let $0<p, s<\infty,-2<q<\infty$ and $0<\alpha<1$. First, we can find a natural metric in $\mathcal{B}_{\alpha}^{*}$ (see [10]) by defining

$$
\mathrm{d}\left(\mathrm{f}, \mathrm{g} ; \mathcal{B}_{\alpha}^{*}\right):=\mathrm{d}_{\mathcal{B}_{\alpha}^{*}}(\mathrm{f}, \mathrm{g})+\|\mathrm{f}-\mathrm{g}\|_{\mathcal{B}_{\alpha}}+|\mathrm{f}(0)-\mathrm{g}(0)|,
$$

where

$$
d_{\mathcal{B}_{\alpha}^{*}}(f, g):=\sup _{z \in \mathbb{D}}\left|\frac{f^{\prime}(z)}{1-|f(z)|^{2}}-\frac{g^{\prime}(z)}{1-|g(z)|^{2}}\right|\left(1-|z|^{2}\right)^{\alpha},
$$

for $f, g \in \mathcal{B}_{\alpha}^{*}$. The presence of the conventional $\alpha$-Bloch-norm here perhaps unexpected. It is motivated by example (see [10, Example in Section 7 ). It shows the phenomenon that, though trivially $d_{\mathcal{B}_{\alpha}^{*}}(f, 0) \geq\|f\|_{\mathcal{B}_{\alpha}}$ for all $f \in \mathcal{B}_{\alpha}^{*}$, the same does no more hold for the differences of two functions: there does not even exist a constant $C>0$ such that

$$
\sup _{z \in \mathbb{D}}\left|\frac{f^{\prime}(z)}{1-|f(z)|^{2}}-\frac{g^{\prime}(z)}{1-|g(z)|^{2}}\right|\left(1-|z|^{2}\right)^{\alpha} \geq C\|f-g\|_{\mathcal{B}_{\alpha}}
$$

would hold for all $\mathrm{f}, \mathrm{g} \in \mathcal{B}_{\alpha}^{*}, 0<\alpha<1$.

For $f, g \in F^{*}(p, q, s)$, define their distance by

$$
d\left(f, g ; F^{*}(p, q, s)\right):=d_{F *}(f, g)+\|f-g\|_{F(p, q, s)}+|f(0)-g(0)|,
$$

where

$$
d_{F^{*}}(f, g):=\left(\sup _{z \in \mathbb{D}} \int_{\mathbb{D}}\left|\frac{f^{\prime}(z)}{1-|f(z)|^{2}}-\frac{g^{\prime}(z)}{1-|g(z)|^{2}}\right|^{p}\left(1-|z|^{2}\right)^{\mathfrak{q}} g^{s}(z, a) d A(z)\right)^{\frac{1}{p}} .
$$

The following characterization of complete metric space $\mathrm{d}\left(., ., ; \mathcal{B}_{\mu}^{*}\right)$ can be proved as in Proposition 2.1 of [10]. 
Proposition 2.1. The class $\mathcal{B}_{\alpha}^{*}$ equipped with the metric $\mathrm{d}\left(., ; ; \mathcal{B}_{\alpha}^{*}\right)$ is a complete metric space. Moreover, $\mathcal{B}_{\alpha, 0}^{*}$ is a closed (and therefore complete) subspace of $\mathcal{B}_{\alpha}^{*}$.

Now we prove the following proposition

Proposition 2.2. The class $\mathrm{F}^{*}(\mathrm{p}, \mathrm{q}, \mathrm{s})$ equipped with the metric $\mathrm{d}\left(., . ; \mathrm{F}^{*}(\mathrm{p}, \mathrm{q}, \mathrm{s})\right)$ is a complete metric space. Moreover, $\mathrm{F}_{0}^{*}(\mathrm{p}, \mathrm{q}, \mathrm{s})$ is a closed (and therefore complete) subspace of $\mathrm{F}^{*}(\mathrm{p}, \mathrm{q}, \mathrm{s})$.

Proof. For $f, g, h \in F^{*}(p, q, s)$, then clearly

- $d\left(f, g ; F^{*}(p, q, s)\right) \geq 0$,

- $d\left(f, f ; F^{*}(p, q, s)\right)=0$,

- $d\left(f, g ; F^{*}(p, q, s)\right)=0$ implies $f=g$.

- $d\left(f, g ; F^{*}(p, q, s)\right)=d\left(g, f ; F^{*}(p, q, s)\right)$

- $d\left(f, h ; F^{*}(p, q, s)\right) \leq d\left(f, g ; F^{*}(p, q, s)\right)+d\left(g, h ; F^{*}(p, q, s)\right)$.

Hence, $d$ is metric on $F^{*}(p, q, s)$.

For the completeness proof, let $\left(f_{n}\right)_{n=0}^{\infty}$ be a Cauchy sequence in the metric space $F^{*}(p, q, s)$, that is, for any $\varepsilon>0$ there is an $N=N(\varepsilon) \in \mathbb{N}$ such that $d\left(f_{n}, f_{m}\right)<\varepsilon$, for all $n, m>N$. Since $f_{n} \in B(\mathbb{D})$ such that $f_{n}$ converges to $f$ uniformly on compact subsets of $\mathbb{D}$. Let $m>N$ and $0<r<1$. Then Fatou's lemma yields

$$
\begin{aligned}
& \int_{\mathbb{D}(0, r)}\left|\frac{f^{\prime}(z)}{1-|f(z)|^{2}}-\frac{f_{m}^{\prime}(z)}{1-\left|f_{m}(z)\right|^{2}}\right|^{p}\left(1-|z|^{2}\right)^{\mathrm{q}} g^{s}(z, a) d A(z) \\
= & \int_{\mathbb{D}(0, r)} \lim _{n \rightarrow \infty}\left|\frac{f_{n}^{\prime}(z)}{1-\left|f_{n}(z)\right|^{2}}-\frac{f_{m}^{\prime}(z)}{1-\left|f_{m}(z)\right|^{2}}\right|^{p}\left(1-|z|^{2}\right)^{\mathrm{q}} g^{s}(z, a) d A(z) \\
\leq & \lim _{n \rightarrow \infty} \int_{\mathbb{D}}\left|\frac{f_{n}^{\prime}(z)}{1-\left|f_{n}(z)\right|^{2}}-\frac{f_{m}^{\prime}(z)}{1-\left|f_{m}(z)\right|^{2}}\right|^{p}\left(1-|z|^{2}\right)^{\mathrm{q}} g^{s}(z, a) d A(z) \leq \varepsilon^{p} .
\end{aligned}
$$

By letting $r \rightarrow 1^{-}$, it follows from inequalities (2) and $(a+b)^{p} \leq 2^{p}\left(a^{p}+b^{p}\right)$ that

$$
\int_{\mathbb{D}}\left(f^{*}(z)\right)^{p}\left(1-|z|^{2}\right)^{q} g^{s}(z, a) d A(z) \leq 2^{p} \varepsilon^{p}+2^{p} \int_{\mathbb{D}}\left(f_{m}^{*}(z)\right)^{p}\left(1-|z|^{2}\right)^{q} g^{s}(z, a) d A(z) .
$$

This yields

$$
\|f\|_{F^{*}(p, q, s)}^{p} \leq 2^{p} \varepsilon^{p}+2^{p}\left\|f_{m}\right\|_{F^{*}(p, q, s)}^{p},
$$

and thus $f \in F^{*}(p, q, s)$. We also find that $f_{n} \rightarrow f$ with respect to the metric of $F^{*}(p, q, s)$.

The second part of the assertion follows by (3). 


\section{Compactness of $C_{\phi}$ in hyperbolic classes}

For $0<p, s<\infty,-2<\mathrm{q}<\infty$ and $0<\alpha<\infty$. We define the following notations:

$$
\Phi_{\phi}(p, q, s, a)=\int_{\mathbb{D}} \frac{\left|\phi^{\prime}(z)\right|^{p}}{\left(1-|\phi(z)|^{2}\right)^{\alpha p}}\left(1-|z|^{2}\right)^{q} g^{s}(z, a) d A(z)
$$

and

$$
\Omega_{\phi, r}(p, q, s, a)=\int_{|\phi| \geq r} \frac{\left|\phi^{\prime}(z)\right|^{p}}{\left(1-|\phi(z)|^{2}\right)^{\alpha p}}\left(1-|z|^{2}\right)^{q} g^{s}(z, a) d A(z) .
$$

Theorem 3.1. Assume $\phi$ is a holomorphic mapping from $\mathbb{D}$ into itself. Let $0 \leq p<\infty, 0 \leq s \leq 1$, $-1<\mathrm{q}<\infty$ and $0<\alpha \leq 1$. Then the following are equivalent:

(i) $\mathrm{C}_{\phi}: \mathcal{B}_{\alpha}^{*} \rightarrow \mathrm{F}^{*}(\mathrm{p}, \mathrm{q}, \mathrm{s})$ is bounded;

(ii) $\mathrm{C}_{\phi}: \mathcal{B}_{\alpha}^{*} \rightarrow \mathrm{F}^{*}(\mathrm{p}, \mathrm{q}, \mathrm{s})$ is Lipschitz continuous;

(iii) $\sup _{a \in \mathbb{D}} \Phi_{\phi}(p, q, s, a)<\infty$.

Proof. First, assume that (i) holds, then there exists a constant C such that

$$
\left\|C_{\phi} f\right\|_{F^{*}(p, q, s)} \leq C\|f\|_{\mathcal{B}_{\alpha}^{*}}, \quad \text { for all } f \in \mathcal{B}_{\alpha}^{*}
$$

For given $f \in \mathcal{B}_{\alpha}^{*}$, the function $f_{t}(z)=f(t z)$, where $0<t<1$, belongs to $\mathcal{B}_{\alpha}^{*}$ with the property $\left\|f_{t}\right\|_{\mathcal{B}_{\alpha}^{*}} \leq\|f\|_{\mathcal{B}_{\alpha}^{*}}$. Let $f, g$ be the functions from Lemma 1.4, such that

$$
\frac{1}{\left(1-|z|^{2}\right)^{\alpha}} \leq f^{*}(z)+g^{*}(z)
$$

for all $z \in \mathbb{D}$, so that

$$
\frac{\left|\phi^{\prime}(z)\right|}{(1-|\phi(z)|)^{\alpha}} \leq(f \circ \phi)^{*}(z)+(g \circ \phi)^{*}(z)
$$

Thus, the inequalities

$$
\begin{aligned}
& \int_{\mathbb{D}} \frac{\left|t \phi^{\prime}(z)\right|^{p}}{\left(1-|t \phi(z)|^{2}\right)^{\alpha p}}\left(1-|z|^{2}\right)^{\mathrm{q}} g^{\mathrm{s}}(z, a) d A(z) \\
\leq & 2^{p} \int_{\mathbb{D}}\left[\left((f \circ t \phi)^{*}(z)\right)^{p}+\left((g \circ t \phi)^{*}(z)\right)^{p}\right]\left(1-|z|^{2}\right)^{\mathrm{q}} g^{\mathrm{s}}(z, a) d A(z) \\
\leq & 2^{p}\left\|C_{\phi}\right\|^{p}\left(\|f\|_{\mathcal{B}_{\alpha}^{*}}^{\mathrm{p}}+\|g\|_{\mathcal{B}_{\alpha}^{*}}^{\mathrm{p}}\right) .
\end{aligned}
$$

This estimate together with the Fatou's lemma implies (iii). 
Conversely, assuming that (iii) holds and that $\mathrm{f} \in \mathcal{B}_{\alpha}^{*}$, we see that

$$
\begin{aligned}
& \left.\sup _{a \in \mathbb{D}} \int_{\mathbb{D}}\left((f \circ \phi)^{*}(z)\right)^{\mathrm{p}}\left(1-|z|^{2}\right)^{\mathrm{q}} g^{\mathrm{s}}(z, a) d A(z)\right) \\
= & \sup _{a \in \mathbb{D}} \int_{\mathbb{D}}\left(f^{*}(\phi(z))\right)^{\mathrm{p}}\left|\phi^{\prime}(z)\right|^{\mathrm{p}}\left(1-|z|^{2}\right)^{\mathrm{q}} g^{\mathrm{s}}(z, a) d A(z) \\
\leq & \|f\|_{\mathcal{B}_{\alpha}^{*}}^{p} \sup _{a \in \mathbb{D}} \int_{\mathbb{D}} \frac{\left|\phi^{\prime}(z)\right|^{\mathrm{p}}}{\left(1-|\phi(z)|^{2}\right)^{\alpha p}}\left(1-|z|^{2}\right)^{\mathrm{q}} g^{\mathrm{s}}(z, a) \mathrm{dA}(z) .
\end{aligned}
$$

Hence, it follows that (i) holds.

(ii) $\Longleftrightarrow$ (iii). Assume first that $\mathrm{C}_{\phi}: \mathcal{B}_{\alpha}^{*} \rightarrow \mathrm{F}^{*}(\mathrm{p}, \mathrm{q}, \mathrm{s})$ is Lipschitz continuous, that is, there exists a positive constant $\mathrm{C}$ such that

$$
d\left(f \circ \phi, g \circ \phi ; F^{*}(p, q, s)\right) \leq C d\left(f, g ; \mathcal{B}_{\alpha}^{*}\right), \quad \text { for all } f, g \in \mathcal{B}_{\alpha}^{*}
$$

Taking $\mathrm{g}=0$, this implies

$$
\|f \circ \phi\|_{F^{*}(p, q, s)} \leq \mathrm{C}\left(\|f\|_{\mathcal{B}_{\alpha}^{*}}+\|f\|_{\mathcal{B}_{\alpha}}+|f(0)|\right), \quad \text { for all } f \in \mathcal{B}_{\alpha}^{*}
$$

The assertion (iii) for $\alpha=1$ follows by choosing $f(z)=z$ in (4). If $0<\alpha<1$, then

$$
\begin{aligned}
|f(z)| & =\left|\int_{0}^{z} f^{\prime}(s) d s+f(0)\right| \leq\|f\|_{\mathcal{B}_{\alpha}} \int_{0}^{|z|} \frac{d x}{\left(1-x^{2}\right)^{\alpha}}+|f(0)| \\
& \leq \frac{\|f\|_{\mathcal{B}_{\alpha}}}{(1-\alpha)}+|f(0)|
\end{aligned}
$$

and $|\mathrm{f}(z)| \leq \tanh ^{-1}(|z|)\|\mathbf{f}\|_{\mathcal{B}_{1}}+|\mathbf{f}(0)|$, where $\tanh ^{-1}$ (.) stands for inverse hyperbolic tangent function. Then, for $0<\alpha<1$, we deduce that

$$
|f(\phi(0))-g(\phi(0))| \leq \frac{\|f-g\|_{\mathcal{B}_{\alpha}}}{(1-\alpha)}+|f(0)-g(0)|
$$

Moreover, Lemma 1.4 implies the existence of $\mathrm{f}, \mathrm{g} \in \mathcal{B}_{\alpha}^{*}$ such that

$$
\left(f^{\prime}(z)+g^{\prime}(z)\right)\left(1-|z|^{2}\right)^{\alpha} \geq C>0, \quad \text { for all } z \in \mathbb{D} \text {. }
$$

Combining (4) and (6) we obtain

$$
\begin{aligned}
& \|f\|_{\mathcal{B}_{\alpha}^{*}}+\|g\|_{\mathcal{B}_{\alpha}^{*}}+\|f\|_{\mathcal{B}_{\alpha}}+\|g\|_{\mathcal{B}_{\alpha}}+|f(0)|+|g(0)| \\
\geq & C \int_{\mathbb{D}} \frac{\left|\phi^{\prime}(z)\right|^{p}}{\left(1-|\phi(z)|^{2}\right)^{\alpha p}}\left(1-|z|^{2}\right)^{q} g^{s}(z, a) d A(z) \\
\geq & C \Phi_{\phi}(\alpha, p, q, s, a),
\end{aligned}
$$

for which the assertion (iii) follows. 
Assume now that (iii) is satisfied, we have from (5) that

$$
\begin{aligned}
& d\left(f \circ \phi, g \circ \phi ; F^{*}(p, q, s)\right)=d_{F^{*}}(f \circ \phi, g \circ \phi)+\|f \circ \phi-g \circ \phi\|_{F(p, q, s)} \\
& +|\mathrm{f}(\phi(0))-\mathrm{g}(\phi(0))| \\
& \leq d_{\mathcal{B}_{\alpha}^{*}}(f, g)\left(\sup _{a \in \mathbb{D}} \int_{\mathbb{D}} \frac{\left|\phi^{\prime}(z)\right|^{p}}{\left(1-|\phi(z)|^{2}\right)^{\alpha p}}\left(1-|z|^{2}\right)^{q} g^{s}(z, a) d A(z)\right)^{\frac{1}{p}} \\
& +\|f-g\|_{\mathcal{B}_{\alpha}}\left(\sup _{a \in \mathbb{D}} \int_{\mathbb{D}} \frac{\left|\phi^{\prime}(z)\right|^{p}}{\left(1-|\phi(z)|^{2}\right)^{\alpha p}}\left(1-|z|^{2}\right)^{q} g^{s}(z, a) d A(z)\right)^{\frac{1}{p}} \\
& +\frac{\|f-g\|_{\mathcal{B}_{\alpha}}}{(1-\alpha)}+|f(0)-g(0)| \\
& \leq \mathrm{C}^{\prime} \mathrm{d}\left(\mathrm{f}, \mathrm{g} ; \mathcal{B}_{\alpha}^{*}\right) \text {. }
\end{aligned}
$$

Thus $\mathrm{C}_{\phi}: \mathcal{B}_{\alpha}^{*} \rightarrow \mathrm{F}^{*}(\mathrm{p}, \mathrm{q}, \mathrm{s})$ is Lipschitz continuous and the proof is completed.

Remark 3.2. Theorem 3.1 shows that $\mathrm{C}_{\phi}: \mathcal{B}_{\alpha}^{*} \rightarrow \mathrm{F}^{*}(\mathrm{p}, \mathrm{q}, \mathrm{s})$ is bounded if and only if it is Lipschitz-continuous, that is, if there exists a positive constant $\mathrm{C}$ such that

$$
\mathrm{d}\left(\mathrm{f} \circ \phi, g \circ \phi ; \mathrm{F}^{*}(\mathrm{p}, \mathrm{q}, \mathrm{s})\right) \leq \mathrm{Cd}\left(\mathrm{f}, \mathrm{g} ; \mathcal{B}_{\alpha}^{*}\right), \quad \text { for all } \mathrm{f}, \mathrm{g} \in \mathcal{B}_{\alpha}^{*}
$$

By elementary functional analysis, a linear operator between normed spaces is bounded if and only if it is continuous, and the boundedness is trivially also equivalent to the Lipschitz-continuity. So, our result for composition operators in hyperbolic spaces is the correct and natural generalization of the linear operator theory.

The following observation is sometimes useful.

Proposition 3.3. Assume $\phi$ is a holomorphic mapping from $\mathbb{D}$ into itself. Let $0<p, s<\infty$, $-1<\mathrm{q}<\infty$ and $0<\alpha<\infty$. If $\mathrm{C}_{\phi}: \mathcal{B}_{\alpha}^{*} \rightarrow \mathrm{F}^{*}(\mathrm{p}, \mathrm{q}, \mathrm{s})$ is compact, it maps closed balls onto compact sets.

Proof. If $\mathrm{B} \subset \mathcal{B}_{\alpha}^{*}$ is a closed ball and $\mathrm{g} \in \mathrm{F}^{*}(\mathrm{p}, \mathrm{q}, \mathrm{s})$ belongs to the closure of $\mathrm{C}_{\phi}(\mathrm{B})$, we can find a sequence $\left(f_{n}\right)_{n=1}^{\infty} \subset B$ such that $f_{n} \circ \phi$ converges to $g \in F^{*}(p, q, s)$ as $n \rightarrow \infty$. But $\left(f_{n}\right)_{n=1}^{\infty}$ is a normal family, hence it has a subsequence $\left(f_{n_{j}}\right)_{j=1}^{\infty}$ converging uniformly on the compact subsets of $\mathbb{D}$ to an analytic function $\mathrm{f}$. As in earlier arguments of Proposition 2.1 in [10], we get a positive estimate which shows that $f$ must belong to the closed ball B. On the other hand, also the sequence $\left(f_{n_{j}} \circ \phi\right)_{j=1}^{\infty}$ converges uniformly on compact subsets to an analytic function, which is $g \in F^{*}(p, q, s)$. We get $g=f \circ \phi$, i.e. $g$ belongs to $C_{\phi}(B)$. Thus, this set is closed and also compact.

Compactness of composition operators can be characterized in full analogy with the linear case. 
Theorem 3.4. Assume $\phi$ is a holomorphic mapping from $\mathbb{D}$ into itself. Let $0<p<\infty,-1<$ $\mathrm{q}<\infty, 0 \leq \mathrm{s} \leq 1$ and $0<\alpha \leq 1$. Then the following are equivalent:

(i) $\mathrm{C}_{\phi}: \mathcal{B}_{\alpha}^{*} \rightarrow \mathrm{F}^{*}(\mathrm{p}, \mathrm{q}, \mathrm{s})$ is compact;

(ii) $\lim _{r \rightarrow 1^{-}} \sup _{a \in \mathbb{D}} \Omega_{\phi, r}(p, q, s, a)=0$.

Proof. We first assume that (ii) holds. Let $\mathrm{B}:=\overline{\mathrm{B}}(\mathrm{g}, \delta) \subset \mathcal{B}_{\alpha}^{*}$, where $\mathrm{g} \in \mathcal{B}_{\alpha}^{*}$ and $\delta>0$, be a closed ball, and let $\left(f_{n}\right)_{n=1}^{\infty} \subset B$ be any sequence. We show that its image has a convergent subsequence in $F^{*}(p, q, s)$, which proves the compactness of $C_{\phi}$ by definition.

Again, $\left(f_{n}\right)_{n=1}^{\infty} \subset B(\mathbb{D})$ implies that, there is a subsequence $\left(f_{n_{j}}\right)_{j=1}^{\infty}$ which converges uniformly on the compact subsets of $\mathbb{D}$ to an analytic function $f$. By the Cauchy formula for the derivative of an analytic function, also the sequence $\left(f_{n_{j}}^{\prime}\right)_{j=1}^{\infty}$ converges uniformly on compact subsets of $\mathbb{D}$ to $f^{\prime}$. It follows that also the sequences $\left(f_{n_{j}} \circ \phi\right)_{j=1}^{\infty}$ and $\left(f_{n_{j}}^{\prime} \circ \phi\right)_{j=1}^{\infty}$ converge uniformly on compact subsets of $\mathbb{D}$ to $f \circ \phi$ and $f^{\prime} \circ \phi$, respectively. Moreover, $f \in B \subset \mathcal{B}_{\alpha}^{*}$ since for any fixed $R, 0<R<1$, the uniform convergence yield $\mathrm{d}\left(\mathrm{f}, \mathrm{g} ; \mathcal{B}_{\alpha}^{*}\right) \leq \delta$ (see [10] pp.130).

Let $\varepsilon>0$. Since (ii) is satisfied, we may fix $r, 0<r<1$, such that

$$
\sup _{a \in \mathbb{D}} \int_{|\phi(z)| \geq r} \frac{|\phi(z)|^{p}}{\left(1-|\phi(z)|^{2}\right)^{\alpha p}}\left(1-|z|^{2}\right)^{\mathrm{q}} g^{\mathrm{s}}(z, a) d A(z) \leq \varepsilon .
$$

By the uniform convergence, we may fix $N_{1} \in \mathbb{N}$ such that

$$
\left|f_{n_{j}} \circ \phi(0)-f \circ \phi(0)\right| \leq \varepsilon, \quad \text { for all } j \geq N_{1} .
$$

The condition (ii) is known to imply the compactness of $\mathrm{C}_{\phi}: \mathcal{B}_{\alpha} \rightarrow \mathrm{F}(\mathrm{p}, \mathrm{q}, \mathrm{s})$, hence, possibly to passing once more to a subsequence and adjusting the notations, we may assume that

$$
\left\|f_{n_{j}} \circ \phi-f \circ \phi\right\|_{F(p, q, s)} \leq \varepsilon, \quad \text { for all } j \geq N_{2} \text {, for some } N_{2} \in \mathbb{N} \text {. }
$$

Now let

$$
I_{1}(a, r)=\sup _{a \in \mathbb{D}} \int_{|\phi(z)| \geq r}\left[\left(f_{n_{j}} \circ \phi\right)^{*}(z)-(g \circ \phi)^{*}(z)\right]^{p}\left(1-|z|^{2}\right)^{q} g^{s}(z, a) d A(z),
$$

and

$$
I_{2}(a, r)=\sup _{a \in \mathbb{D}} \int_{|\phi(z)| \leq r}\left[\left(f_{n_{j}} \circ \phi\right)^{*}(z)-(g \circ \phi)^{*}(z)\right]^{p}\left(1-|z|^{2}\right)^{q} g^{s}(z, a) d A(z) .
$$


Since $\left(f_{n_{j}}\right)_{j=1}^{\infty} \subset B$ and $f \in B$, it follows from (1) that

$$
\begin{aligned}
I_{1}(a, r) & =\sup _{a \in \mathbb{D}} \int_{|\phi(z)| \geq r}\left[\left(f_{n_{j}} \circ \phi\right)^{*}(z)-(g \circ \phi)^{*}(z)\right]^{p}\left(1-|z|^{2}\right)^{q} g^{s}(z, a) d A(z) \\
& \leq \sup _{a \in \mathbb{D}} \int_{|\phi(z)| \geq r}\left|\frac{\left(f_{n_{j}} \circ \phi\right)^{\prime}(z)}{1-\left|\left(f_{n_{j}} \circ \phi\right)(z)\right|^{2}}-\frac{(g \circ \phi)^{\prime}(z)}{1-|(g \circ \phi)(z)|^{2}}\right|^{p}\left(1-|z|^{2}\right)^{q} g^{s}(z, a) d A(z) \\
& =\sup _{a \in \mathbb{D}} \int_{|\phi(z)| \geq r} M\left(f_{n_{j}}, g, \phi ; \alpha, p\right)\left(1-|z|^{2}\right)^{q} g^{s}(z, a) d A(z) \\
& \leq d_{\mathcal{B}_{\alpha}^{*}}\left(f_{n_{j}}, f\right) \sup _{a \in \mathbb{D}} \int_{|\phi(z)| \geq r} \frac{|\phi(z)|^{p}}{\left(1-|\phi(z)|^{2}\right)^{\alpha p}}\left(1-|z|^{2}\right)^{q} g^{s}(z, a) d A(z),
\end{aligned}
$$

where

$$
M\left(f_{n_{j}}, g, \phi ; \alpha, p\right)=\left|\left(\frac{f_{n_{j}}^{\prime}(\phi(z))}{1-\left|f_{n_{j}}(\phi(z))\right|^{2}}-\frac{g^{\prime}(\phi(z))}{1-\mid g\left(\left(\left.\phi(z)\right|^{2}\right.\right.}\right)\left(1-|\phi(z)|^{2}\right)^{\alpha}\right|^{p}\left|\frac{\phi^{\prime}(z)}{\left(1-|\phi(z)|^{2}\right)^{\alpha}}\right|^{p} .
$$

Hence,

$$
I_{1}(a, r) \leq 2 \delta \varepsilon
$$

On the other hand, by the uniform convergence on compact subsets of $\mathbb{D}$, we can find an $N_{3} \in \mathbb{N}$ such that for all $j \geq N_{3}$,

$$
\left|\frac{f_{n_{j}}^{\prime}(\phi(z))}{1-\left|f_{n_{j}}(\phi(z))\right|^{2}}-\frac{f^{\prime}(\phi(z))}{1-|f(\phi(z))|^{2}}\right| \leq \varepsilon
$$

for all $z$ with $|\phi(z)| \leq r$. Hence, for such $j$,

$$
\begin{aligned}
\mathrm{I}_{2}(\mathrm{a}, \mathrm{r}) & =\sup _{\mathrm{a} \in \mathbb{D}} \int_{|\phi(z)| \leq \mathrm{r}}\left[\left(f_{n_{j}} \circ \phi\right)^{*}(z)-(g \circ \phi)^{*}(z)\right]^{\mathrm{p}}\left(1-|z|^{2}\right)^{\mathrm{q}} g^{\mathrm{s}}(z, a) d A(z) \\
& \leq \sup _{a \in \mathbb{D}} \int_{|\phi(z)| \leq r}\left|\frac{\left(f_{n_{j}} \circ \phi\right)^{\prime}(z)}{1-\left|\left(f_{n_{j}} \circ \phi\right)(z)\right|^{2}}-\frac{(g \circ \phi)^{\prime}(z)}{1-|(g \circ \phi)(z)|^{2}}\right|^{p}\left(1-|z|^{2}\right)^{\mathrm{q}} g^{\mathrm{s}}(z, a) d A(z) \\
& \leq \varepsilon\left(\sup _{a \in \mathbb{D}} \int_{|\phi(z)| \leq r} \frac{|\phi(z)|^{p}}{\left(1-|\phi(z)|^{2}\right)^{\alpha p}}\left(1-|z|^{2}\right)^{\mathrm{q}} g^{\mathrm{s}}(z, a) d A(z)\right)^{\frac{1}{p}} \leq \mathrm{C} \varepsilon
\end{aligned}
$$

hence,

$$
\mathrm{I}_{2}(\mathrm{a}, \mathrm{r}) \leq \mathrm{C} \varepsilon
$$

where $C$ is the bounded obtained from (iii) of Theorem [3.1. Combining (7), (8), (9) and (10) we deduce that $f_{n_{j}} \rightarrow f$ in $F^{*}(p, q, s)$.

As for the converse direction, let $f_{n}(z):=\frac{1}{2} n^{\alpha-1} z^{n}$ for all $n \in N, n \geq 2$. Then the sequence $\left(f_{n}\right)_{n=1}^{\infty}$ belongs to the ball $\bar{B}(0,3) \subset \mathcal{B}_{\alpha}^{*}($ see [10] pp.131).

We are assuming that $C_{\phi}$ maps the closed ball $\bar{B}(0,3) \subset \mathcal{B}_{\alpha}^{*}$ into a compact subset of $F^{*}(p, q, s)$, hence, there exists an unbounded increasing subsequence $\left(f_{n_{j}}\right)_{j=1}^{\infty}$ such that the image subsequence $\left(C_{\phi} f_{n_{j}}\right)_{j=1}^{\infty}$ converges with respect to the norm. Since, both $\left(f_{n}\right)_{n=1}^{\infty}$ and $\left(C_{\phi} f_{n_{j}}\right)_{j=1}^{\infty}$ converge to 
the zero function uniformly on compact subsets of $\mathbb{D}$, the limit of the latter sequence must be 0 . Hence,

$$
\left\|n_{j}^{\alpha-1} \phi^{n_{j}}\right\|_{F^{*}(p, q, s)} \rightarrow 0, \quad \text { as } j \rightarrow \infty .
$$

Now let $r_{j}=1-\frac{1}{n_{j}}$. For all numbers $a, r_{j} \leq a<1$, we have the estimate (see [10])

$$
\frac{n_{j}^{\alpha} a^{n_{j}-1}}{1-a^{n_{j}}} \geq \frac{1}{e(1-a)^{\alpha}}
$$

Using (12) we obtain

$$
\begin{aligned}
\left\|n_{j}^{\alpha-1} \phi^{n_{j}}\right\|_{F^{*}(p, q, s)}^{p} & \geq \sup _{a \in \mathbb{D}} \int_{|\phi| \geq r_{j}}\left|\frac{n_{j}^{\alpha}(\phi(z))^{n_{j}-1} \phi^{\prime}(z)}{1-\left|\phi^{n_{j}}(z)\right|^{2}}\right|^{p}\left(1-|z|^{2}\right)^{q} g^{s}(z, a) d A(z) \\
& \geq \frac{1}{(2 e)^{p}} \sup _{a \in \mathbb{D}} \int_{|\phi| \geq r_{j}} \frac{\left|\phi^{\prime}(z)\right|^{p}}{\left(1-\mid \phi(z)^{2}\right)^{\alpha p}}\left(1-|z|^{2}\right)^{q} g^{s}(z, a) d A(z) .
\end{aligned}
$$

Hence, the condition (ii) follows.

Received: February 2012. Accepted: November 2012.

\section{References}

[1] A. El-Sayed Ahmed, Natural metrics and composition operators in generalized hyperbolic function spaces, Journal of inequalities and applications, 185(2012), 1-12.

[2] A. El-Sayed Ahmed and M. A. Bakhit, Composition operators on some holomorphic Banach function spaces, Mathematica Scandinavica, 104(2)(2009), 275-295.

[3] A. El-Sayed Ahmed and M. A. Bakhit, Composition operators acting between some weighted Möbius invariant spaces, Ann. Funct. Anal. AFA 2(2)(2011), 138-152.

[4] C. Cowen and B. D. MacCluer, Composition operators on spaces of analytic functions, Studies in Advanced Mathematics. Boca Raton, FL: CRC Press. xii, 1995.

[5] M. Kotilainen, Studies on composition operators and function spaces, Report Series. Department of Mathematics, University of Joensuu 11. (Dissertation) 2007.

[6] P. Lappan and J. Xiao, $\mathcal{Q}_{\alpha}^{\#}$-bounded composition maps on normal classes, Note di Matematica, 20(1) (2000/2001), 65-72.

[7] X. Li, On hyperbolic Q classes, Dissertation, University of Joensuu, Joensuu, 2005, Ann. Acad. Sci. Fenn. Math. Diss. 145 (2005), 65 pp.

[8] X. Li, F. Pérez-González, and J. Rättyä, Composition operators in hyperbolic Q-classes, Ann. Acad. Sci. Fenn. Math. 31 (2006), 391-404. 
[9] S. Makhmutov and M. Tjani, Composition operators on some Möbius invariant Banach spaces, Bull. Austral. Math. Soc. 62 (2000), 1-19.

[10] F. Pérez-González, J. Rättyä and J. Taskinen, Lipschitz Continuous and Compact Composition Operators in Hyperbolic Classes, Mediterr. J. Math. 8 (2011), 123-135.

[11] W. Smith, Inner functions in the hyperbolic little Bloch class, Michigan Math. J. 45(1) (1998), 103-114.

[12] M. Tjani, Compact composition operators on Besov spaces, Trans. Amer. Math. Soc. 355 (2003), 4683-4698.

[13] J. Xiao, Holomorphic Q classes, Lecture Notes in Mathematics, 1767, Springer-Verlag, Berlin, 2001.

[14] S. Yamashita, Hyperbolic Hardy classes and hyperbolically Dirichlet-finite functions, Hokkaido Math. J., Special Issue 10 (1981), 709-722.

[15] S. Yamashita, Functions with $\mathrm{H}^{\mathrm{p}}$ hyperbolic derivative, Math. Scand. 53 (2)(1983), 238-244.

[16] S. Yamashita, Holomorphic functions of hyperbolic bounded mean oscillation, Boll. Un. Math. Ital. 5 B(6), (3)(1986), 983-1000.

[17] J. Zhou, Composition operators from $\mathcal{B}^{\alpha}$ to $\mathcal{Q}_{\mathrm{K}}$ type spaces, J. Funct. Spaces Appl. 6 (1)(2008), 89-105. 